\section{Mini-Review}

Correspondence

Sven Hammerschmidt

s.hammerschmidt@mail.uni-

wuerzburg.de

\title{
Versatility of pneumococcal surface proteins
}

\author{
Simone Bergmann and Sven Hammerschmidt \\ Research Center for Infectious Diseases, University of Würzburg, Röntgenring 11, D-97070 \\ Würzburg, Germany
}

\begin{abstract}
Surface-exposed proteins are key players during the infectious process of pathogenic bacteria. The cell surface of the Gram-positive human pathogen Streptococcus pneumoniae is decorated not only by typical Gram-positive surface proteins, but also by a family of proteins that recognizes the phosphorylcholine of the lipoteichoic and teichoic acids, namely the choline-binding proteins, and by non-classical surface proteins that lack a leader peptide and membrane-anchor motif. A comprehensive understanding of how microbial proteins subvert host immunity or host protein functions is a prerequisite for the development of novel therapeutic strategies to combat pneumococcal infections. This article reviews recent progress in the investigation of the versatility and sophistication of the virulence functions of surface-exposed pneumococcal proteins.
\end{abstract}

\section{The power of combinatorial diversity}

Streptococcus pneumoniae (the pneumococcus) is a commensal of the nasopharyngeal cavity, but under appropriate conditions, pneumococci cause serious and life-threatening infections, including pneumonia, septicaemia and meningitis (Cartwright, 2002). Pneumococci are encased by a capsular polysaccharide which has been recognized as a sine qua non of virulence. On the other hand, several studies have indicated that high amounts of capsular polysaccharide prevent attachment to host cells, probably by masking underlying virulence determinants. Interestingly, the amount of capsule is substantially reduced upon contact with epithelial cells (Hammerschmidt et al., 2005). The role of the capsule in immune evasion and the impact of the virulence factor pneumolysin, which represents a cholesterol-dependent cytolysin, on pathogenesis are reviewed by Paterson \& Mitchell (2006) in this issue. The pneumococcal outer cell wall is composed of peptidoglycan, teichoic (TA) and lipoteichoic acids (LTA), which differ only in their attachment to the pneumococcal cell wall, and phosphorylcholine (PCho). PCho is not only targeted by the choline-binding domain (CBD) of choline-binding proteins (CBP), but functions itself as an adhesin by recognizing the plateletactivating factor receptor (PAFr) of host cells (Cundell et al., 1995).

Bioinformatic analysis of the genome of S. pneumoniae R6, the rough derivative of serotype 2 strain D39, predicts the presence of 153 proteins with a leader peptide. The leader peptide is recognized by complex secretion machineries known as translocons, and is required for protein traversal across the membranes. In contrast to strain R6, analysis of the sequence of the serotype 4 Norwegian clinical isolate TIGR4 suggests the existence of 256 proteins with a leader peptide (Tettelin \& Hollingshead, 2004). Interestingly, TIGR4 contains two $\sec A$ genes ( $\sec A 1$ and $\sec A 2)$, whereas R6 and probably many other pneumococci contain only secAl. SecA is the main factor of the general secretory pathway, and the ATPase activity of this protein is the molecular motor of protein translocation across the membranes. SecA2 is also present in other Gram-positive bacteria, and has been characterized in Streptococcus gordonii strain M99, in which it is thought to be part of a specialized system for the transport of large serine-rich repeat proteins (Bensing \& Sullam, 2002; Takamatsu et al., 2004).

Three clusters of surface proteins can be distinguished by genome analysis: the lipoproteins (42 in R6 and 47 in TIGR4), the CBP family (10 in R6 and 15 in TIGR4), and proteins with an LPxTG motif (13 in R6 and 19 in TIGR4) that are covalently anchored in the cell wall after cleavage of the LPxTG sequence by a transpeptidase, designated a sortase (Fig. 1). Bioinformatic analysis of the pneumococcal genomes also indicates the presence of incomplete biosynthetic pathways, which is consistent with the inability of this pathogen to carry out respiratory metabolism, and also explains the high number of ATP binding cassette (ABC) transporters produced by $S$. pneumoniae. In addition to these predicted surface proteins, non-classical surface proteins that lack a classical leader peptide and membraneanchoring motifs have been identified on the pneumococcal surface (Fig. 2). These proteins have received considerable attention for their contribution to the virulence of pneumococci and other pathogenic bacteria. Proteome analysis of the secretome and outer-membrane proteins of pneumococci is required to reveal the total composition of these protein fractions and the number of non-classical proteins translocated across the cell wall. To date, the mechanism of secretion and anchoring of these proteins, sometimes known as 'moonlighting' proteins, remains unknown for pneumococci and many other Gram-positive pathogens. 
TIGR4

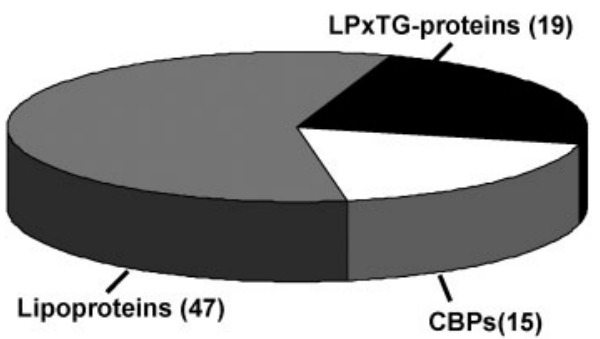

R6

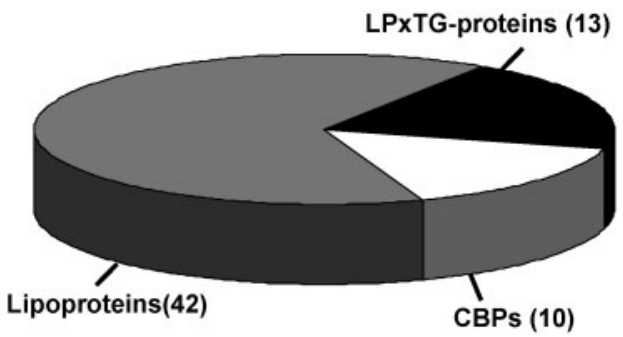

Fig. 1. Proportion of classical pneumococcal surface proteins based on the genome data for strain R6 (the nonencapsulated derivative of serotype 2 strain D39) (right-hand panel) and serotype 4 strain TIGR4 (Tettelin \& Hollingshead, 2004) (left-hand panel). Surface-exposed proteins are distinguished according to their binding mechanism to the cell wall (lipoproteins and LPxTG proteins) or their non-covalent attachment to the cell wall via PCho. Bioinformatic analysis of TIGR4 predicts the presence of 256 proteins carrying a signal peptide for secretion, of which 81 contain classical anchoring motifs. In pneumococcal strain R6, 153 proteins are thought to be secreted, and of these, 65 exhibit classical anchoring motifs. Nonclassical surface-exposed proteins are not considered. The numbers in parentheses show the number of proteins in each class.

\section{Biological activities of unusually cell-wall- anchored choline-binding proteins}

$P C h o$ is an unusual and physiologically important component of the pneumococcal cell wall. The pneumococcal PCho is recognized by components of the host innate immune system, such as C-reactive protein and the PAFr (Fig. 3; Cundell et al., 1995). In addition, the amino alcohol $P C h o$ anchors a special class of pneumococcal proteins, the CBPs, non-covalently to the cell wall. CBPs have a modular organization and consist in general of a leader peptide, a biologically active $\mathrm{N}$-terminal domain, and the conserved CBD that targets PCho. Pneumococci can produce 13 to 16 different CBPs, including four cell wall hydrolases that are important for virulence: the major autolysin LytA ( $N$-acetylmuramoyl-L-alanine amidase), a $\beta$ - $N$-acetylglucosamidase (LytB), a $\beta$-N-acetylmuramidase (LytC; lysozyme), and a phosphorylcholine esterase. LytA has been characterized in detail, and recently the structural analysis of the CBD of LytA demonstrated that this module adopts a peculiar solenoid structure (López \& García, 2004). LytB is highly expressed in the early exponential growth phase and has been shown to be important for cell separation of pneumococci. Loss of function of LytB or LytC significantly reduces the nasopharyngeal colonization of rats (Gosink et al., 2000). In contrast to other CBPs, LytB and LytC possess the CBD as an N-terminal domain (López \& García, 2004).

The phosphorylcholine esterase activity of the Pce protein (also referred to as $\mathrm{CbpE}$ ) removes PCho from the cell wall and causes changes in colony phenotype (de las Rivas et al., 2001; Vollmer \& Tomasz, 2001). Recently, structural information for the catalytic domain (Garau et al., 2005)

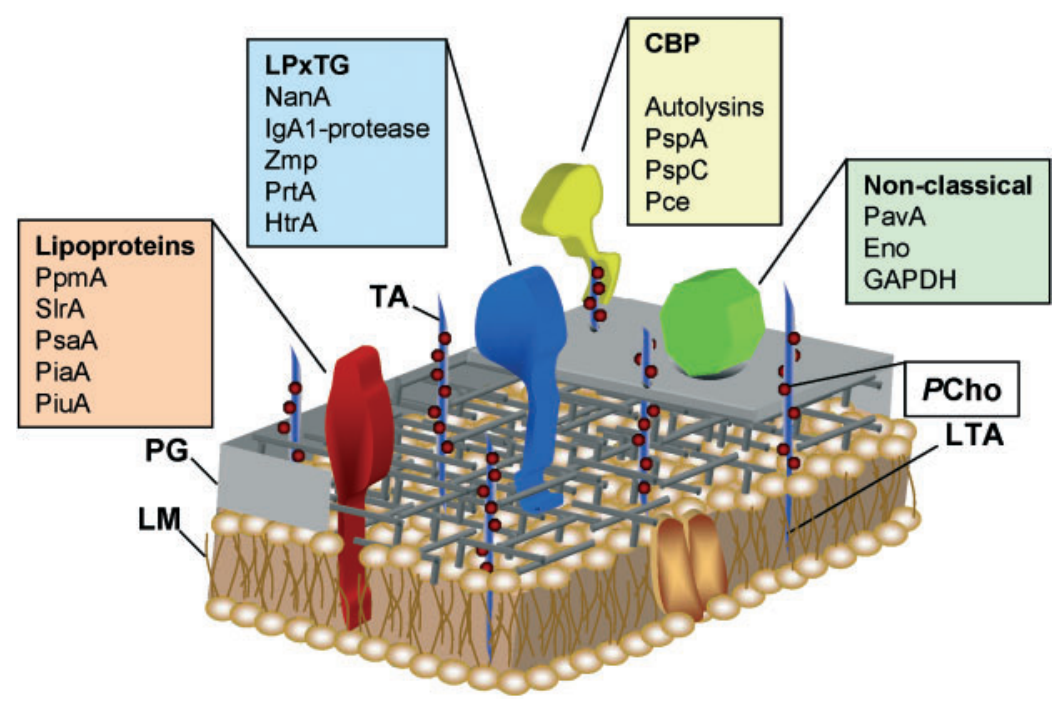

Fig. 2. Schematic model of the pneumococcal outer cell wall and surface-exposed proteins. The pneumococcal cell wall consists of a phospholipid membrane (LM), peptidoglycan (PG), and teichoic and lipoteichoic acids. An unusual component of the cell wall is phophorylcholine (PCho), which anchors the choline-binding proteins (CBPs) non-covalently on the cell wall. Virulence proteins of the different classes of pneumococcal surface proteins are depicted. 


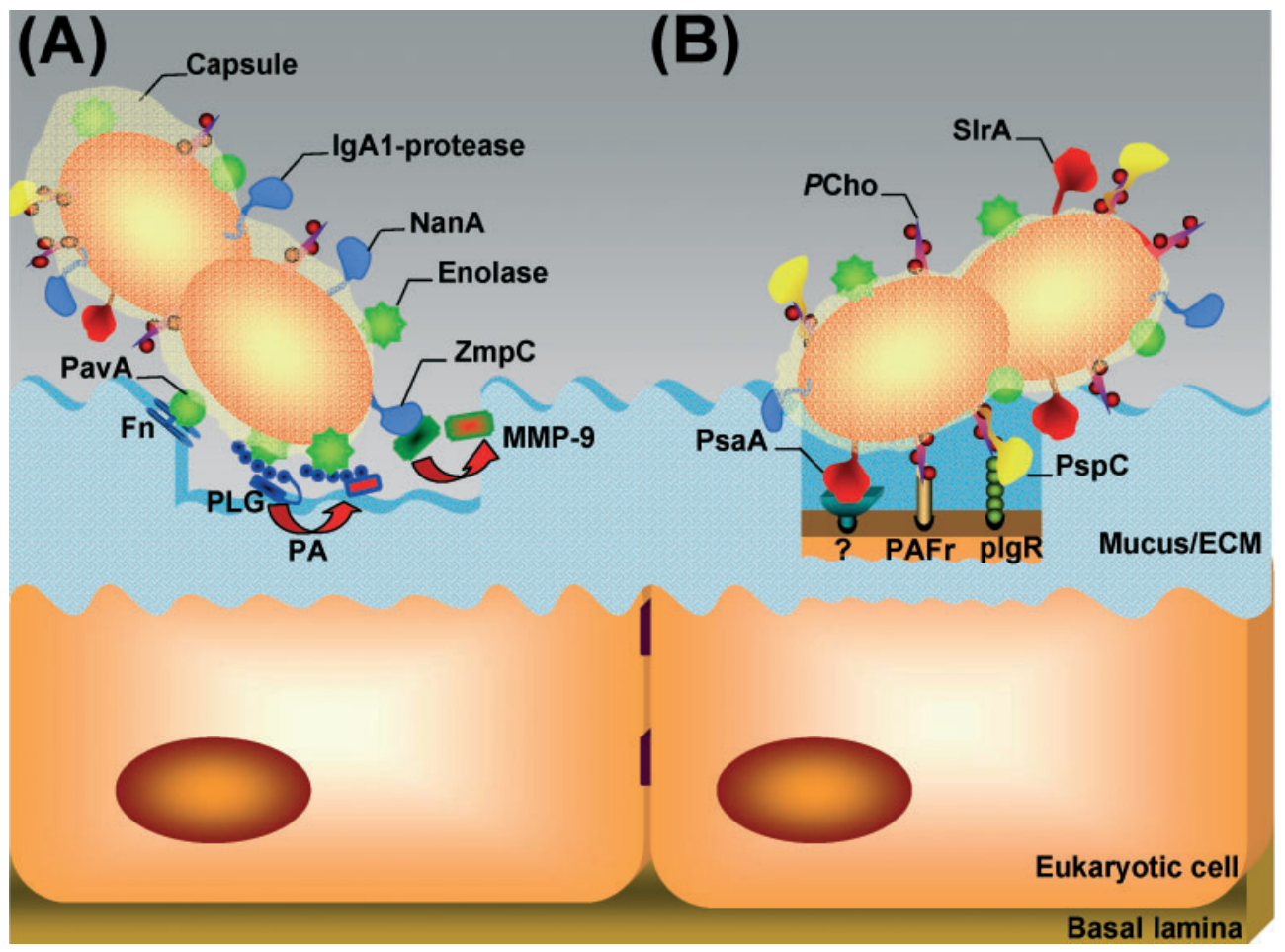

Fig. 3. Interactions of pneumococcal surface proteins with host components of the mucosal cavity $(A)$ and cellular receptors (B). (A) The ECM represents the first mechanical barrier for pneumococcal colonization. Colonization is facilitated by cleavage of bound $\lg \mathrm{A} 1$ by the $\lg \mathrm{A} 1$-protease and by the removal of terminal sialic acids by NanA. The glycolytic enzymes enolase and GAPDH bind human plasminogen (PLG), which is converted to the protease plasmin (PA) and promotes degradation of various ECM compounds. ZmpC activates MMP-9, which has collagenase activity and degrades the ECM. The non-classical surface-associated protein PavA has been shown to bind to immobilized fibronectin $(\mathrm{Fn})$ and to modulate pneumococcal adherence. (B) Degradation of ECM is possibly a prerequisite for the intimate contact of pneumococci with cellular receptors. The major adhesin of pneumococci identified is $\mathrm{PspC}$, which interacts with the ectodomain of the human polymeric immunoglobulin receptor (plgR). PCho mediates pneumococcal adherence to stimulated cells via an interaction with upregulated PAFr. It is thought that the lipoprotein PsaA may also function as an adhesin, but the cellular receptor is not yet defined.

and for the complete Pce lacking only the C-terminal 85residue tail (Hermoso et al., 2005) has been reported. Pce belongs to the metallo- $\beta$-lactamase family, and structural information provides evidence that only PCho residues that are located at the end of the teichoic acid chains are accessible to the catalytic centre. The ability of Pce to modify the amount of PCho on the cell wall has been shown to be relevant for pneumococcal adherence to human cells and for nasopharyngeal colonization of rats (Gosink et al., 2000). Strikingly, loss of function has also been shown to increase the virulence of pneumococci when inoculated into the peritoneum of mice (Vollmer \& Tomasz, 2001). It is possible that the increased number of choline residues promotes interaction with the PAFr during infection. On the other hand, $P$ Cho binds the C-reactive protein, and it has been shown that higher amounts of $P$ Cho favour binding of the host protein. This might lead to enhanced phagocytosis of pneumococci. Moreover, Pce is involved in hydrolysis of the choline-containing platelet-activating factor. In conclusion, Pce may have a dual function and may favour both colonization and invasive infection by modulating the amount of PCho on the pneumococcal cell wall.

A role in colonization has been suggested for $\mathrm{CbpD}$ and $\mathrm{CbpG}$, which are thought to be serine proteases (Gosink et al., 2000). Recent reports indicate that $\mathrm{CbpD}$ is a competence-stimulating-peptide-inducible protein, and a function as a murein hydrolase has been proposed. $\mathrm{CbpD}$ has been demonstrated to assist LytA in competenceinduced cell lysis (Kausmally et al., 2005). A further study has provided experimental evidence that the biological activity of $\mathrm{CbpD}$ is involved in the ability of competent bacteria to trigger the release of virulence factors from noncompetent S. pneumoniae (Guiral et al., 2005).

The highly variable pneumococcal surface protein A (PspA) is expressed by virtually all important clinical serotypes and has significant immune protective potential. Loss of function attenuates virulence and increases complementreceptor-mediated clearance of pneumococci (Ren et al., 2004). PspA also binds the iron transporter lactoferrin 
(Hammerschmidt et al., 1999), but pneumococci are not able to utilize lactoferrin as an iron source. Lactoferrin plays an important role in innate immunity, and the irondepleted form, apolactoferrin, is bacteriostatic or bactericidal for bacteria. Shaper et al. (2004) demonstrated that expression of PspA protects against the bactericidal effect of apolactoferrin. In the presence of antibodies recognizing PspA, this effect is abrogated, suggesting that binding of apolactoferrin to PspA blocks the bactericidal activity of apolactoferrin. Recently it has been shown that the phosphorylated VicR (YycF) response regulator of the twocomponent regulatory system (TCS) VicRK (YycF) binds to a region upstream of $p s p A$ and regulates expression of $p s p A$ (Ng et al., 2005).

The pneumococcal surface protein $\mathrm{C}$ (PspC; also known as SpsA or CbpA) is a multifunctional CBP of pneumococci; PspC promotes uptake of pneumococci into nasopharyngeal epithelial cells and interferes with components of the innate immune system. PspC promotes pneumococcal adherence via a human-specific interaction with the ectodomain of the polymeric Ig receptor (Hammerschmidt et al., 2000; Zhang et al., 2000). The ectodomain of pIgR is also known as the secretory component (SC), and independent reports have indicated that the human specificity of the PspC-SC interaction is determined by amino acid differences in ectodomains D3 and D4 of the SC (Elm et al., 2004; Lu et al., 2003). Despite such human specificity, loss of function in PspC has been shown to reduce colonization of infant rats (Rosenow et al., 1997) and pIgR knockout mice (Zhang et al., 2000). The hexameric SC-binding site in PspC is located in N-terminal repeated domains (Hammerschmidt et al., 2000), recently designated R1 and R2. Structural analysis of R1 (amino acids 175-285) and R2 (amino acids 327-442) of PspC derived from TIGR4 has demonstrated that the R domains adopt an unusual and simple structure composed of three $\alpha$-helices. Bundling of the helices through $\alpha$-helix$\alpha$-helix interactions results in a flat, raft-like structure in which the residues YPT of the minimal SC-binding motif are located in a loop between helix 1 and helix 2 and form a 'tyrosine fork' structure (Luo et al., 2005).

PspC molecules are divided into different groups. Interestingly, both the classical PspC proteins containing a CBD and the PspC-like Hic (PspC 11.4) bind the complement factor H (Janulczyk et al., 2000). Hic is produced predominantly by serotype 3 pneumococcal strains, negative for SC binding, and contains an LPxTG motif that anchors the protein in a sortase-dependent manner to the peptidoglycan backbone of the cell wall. Factor $\mathrm{H}$ is a fluid-phase regulator of the alternative complement pathway, and consists of 20 short consensus repeats (SCRs). Hic interacts with the SCRs $8-11$ and 12-14 of factor H (Jarva et al., 2004), whereas a role for the SCRs $6-10$ and $13-15$ of factor $\mathrm{H}$ has been suggested in the interaction with PspC (Dave et al., 2004; Duthy et al., 2002). Recruitment of factor $\mathrm{H}$ by Hic has been shown to efficiently prevent activation of $\mathrm{C} 3 \mathrm{~b}$ and complement-mediated opsonophagocytosis of pneumococci (Jarva et al., 2004). The improved survival of pneumococci expressing PspC or Hic in a systemic mouse infection model provides further evidence for the versatility and importance of PspC in different host niches (Iannelli et al., 2004; Quin et al., 2005).

\section{The ubiquitous mechanism of Gram-positive protein anchoring to the cell wall peptidoglycan}

Sortase is a transpeptidase that recognizes the LPxTG motif of classical Gram-positive surface proteins and anchors these proteins covalently to the cell wall peptidoglycan. In strain R6 (the nonencapsulated derivative of serotype 2 strain D39), sequence analysis has revealed a single gene that codes for a sortase, whereas four genes $(s r t A, s r t B, s r t C$ and $s r t D)$ are predicted for the TIGR4 strain. The sortase of R6 and srtA of TIGR4 show $99 \%$ identity, and both genes are located downstream of the DNA gyrase-encoding gene (spr1099 and SP1219, respectively). The genes $s r t B$, srtC and $s r t D$ are sequentially organized at a different gene locus. The presence of up to four genes that code for a transpeptidase is interesting, because only a minority of pneumococcal surface proteins are processed by a sortase (Fig. 2). Kharat \& Tomasz (2003) have provided evidence that the sortase A processes functionally different surface-exposed pneumococcal proteins, such as the $\beta$-galactosidase and neuraminidase A. Loss of function in SrtA in a type 3 encapsulated derivative of R36A ( $\mathrm{R} 6$ is a derivative of R36A) has not been associated with impaired virulence in a mouse intraperitoneal model of infection, but has been shown to decrease significantly adherence of R36A or R6 to nasopharyngeal cells (Kharat \& Tomasz, 2003). In serotype 4 strain TIGR4, the srtD knockout significantly impairs pneumococcal pneumonia (Hava \& Camilli, 2002). Recently, Paterson \& Mitchell (2005) have indicated in in vivo studies the contribution of SrtA in pneumococcal pneumonia and bacteraemia. In conclusion, it seems that different sortases process functionally different surface-exposed proteins and have, therefore, different impacts on pneumococcal adherence and virulence.

Pneumococci contain at least two genes, nan $A$ and nanB, that encode neuraminidases, and virtually all strains produce a neuraminidase. A third putative neuraminidase, nan $C$, remains to be characterized. These exoglycosidases cleave terminal sialic acid ( $N$-acetylneuraminic acid) from glycolipids, glycoproteins and oligosaccharides on host cell surfaces and in host body fluids. NanA and NanB are secreted via the general secretory pathway, but only the Cterminus of NanA contains the LPxTG anchoring motif. Loss of NanA has been shown to impair pneumococcal persistence in the nasopharynx and middle ear in a chinchilla infection model (Tong et al., 2002). In contrast, the nanA knockout is not attenuated in an intraperitoneal infection model (Berry \& Paton, 2000). The importance of NanA for colonization has been underlined by its ability to afford protection against nasopharyngeal colonization and otitis media in chinchilla models (Long et al., 2004). Recently, two reports have demonstrated the precise 
function of NanA during pneumococcal colonization and pathogenesis. Firstly, neuraminidase has been shown to cleave the terminal sialic acids of lipooligosaccharides from Haemophilus influenzae and Neisseria meningitidis (Shakhnovich et al., 2002). Because terminal sialic acids protect these respiratory pathogens against complementmediated phagocytosis, desialylation of competitors may provide an advantage during colonization of host niches. Secondly, NanA has been shown to be implicated in desialylation of human proteins exhibiting sialic acid, including the secretory component, lactoferrin and IgA2 (King et al., 2004). These human proteins are pneumococcal host targets, and the removal of sialic acid may facilitate bacterial persistence in the respiratory tract. nanA gene diversity, which occurs by recombination events and is restricted to regions in nanA that are not required for enzymic activity, has been suggested to provide an important advantage in evading the adaptive immune response (King et al., 2005).

The hyaluronate lyase (Hyl), which primarily hydrolyses hyaluronan, is a four-domain enzyme consisting of an $\mathrm{N}$-terminal carbohydrate domain, followed by a spacer domain, the helical barrel-like catalytic domain, and finally the C-terminal domain (Rigden \& Jedrzejas, 2003). hyl knockout mutants are attenuated in an intraperitoneal mouse infection model (Chapuy-Regaud et al., 2003), but the precise function of $\mathrm{Hyl}$ during pathogenesis has yet to be clarified.

\section{Armed with proteolytic activity}

Pneumococci produce several proteases that are surfaceexposed and implicated in pneumococcal virulence. The HtrA (high-temperature requirement A) protease functions in a temperature-dependent manner as a molecular chaperone or heat-shock-induced serine protease, and is regulated by the CiaRH TCS. HtrA has been shown to be implicated in resistance to oxidative stress, in the colonization of the nasopharynx of rats and in pneumococcal pneumonia. Moreover, htrA knockouts induce lower levels of inflammatory cytokines IL- 6 and TNF- $\alpha$ in the lungs during pneumonia compared to the isogenic wild-type D39 (Sebert et al., 2002; Mascher et al., 2003; Ibrahim et al., 2004).

S. pneumoniae strains produce up to four zinc metalloproteases, including IgA1-protease, $\mathrm{ZmpB}, \mathrm{ZmpC}$ and $\mathrm{ZmpD}$, which are anchored to the cell wall by an $\mathrm{N}$-terminal LPxTG motif. The IgA1-protease is produced by virtually all pneumococci, and large-scale signature-tagged mutagenesis (STM) experiments have indicated the importance of the IgA-protease in pneumococcal lung infections and bacteraemia (Polissi et al., 1998). Recently, Weiser et al. (2003) have demonstrated that cleavage of surface-bound serotypespecific IgA1 by the IgA1-protease markedly enhances adherence of pneumococci to host cells. It is assumed that bound Fab fragments neutralize the negatively charged capsule and negate the anti-adhesive effects of the capsule. $\mathrm{ZmpC}$ has been characterized in TIGR4 as a bacterial protease cleaving human matrix metalloproteinase 9 (MMP-9), and inactivation of the $z m p C$ gene in serotype $19 \mathrm{~F}$ impairs virulence in a pneumonia mouse model (Oggioni et al., 2003). MMP-9 is involved in matrix degradation and opening of the blood-brain barrier; therefore, cleavage of the MMP-9 proenzyme to active MMP-9 may promote disease. Intranasal infection experiments confirm the significant contribution of IgA1-protease and $\mathrm{ZmpB}$ to pneumococcal virulence (Blue et al., 2003; Chiavolini et al., 2003). PrtA is another surface-exposed serine protease, and prtA knockouts have been shown to be attenuated in an intraperitoneal mouse infection model (Bethe et al., 2001). It seems obvious that the different proteases may elicit their function at different stages of the pneumococcal infection. To date, which of the proteases act in combination to enhance the virulence of $S$. pneumoniae remains unclear.

\section{Lipoproteins: implications in substrate transport and virulence}

Pneumococcal lipoproteins, including peptide permeases, have been shown to be essential for substrate transport and bacterial fitness. The pneumococcal surface adhesin A (PsaA) is the substrate-binding lipoprotein of an ABC-type manganese-transport system (Dintilhac et al., 1997). Mutations in $p s a A$ cause pleiotropic effects, including reduced adherence of pneumococci to host cells, attenuation in an intranasal and intraperitoneal mouse infection model, and increased sensitivity to oxidative stress (Marra et al., 2002; Tseng et al., 2002). Since the detection of these pleiotropic effects, it has been assumed that PsaA does not itself function as an adhesin; however, a recent report has demonstrated that antibodies against PsaA reduce the adherence of pneumococci to nasopharyngeal epithelial cells (RomeroSteiner et al., 2003). This is consistent with the finding that mucosal immunization of mice with PsaA is highly protective against pneumococcal carriage (Johnson et al., 2002). Differential fluorescence induction (DIF) analysis has demonstrated the upregulation of the $p s a$ promoter of the $p s a B C A$ operon during lung infections, and microarray analysis has demonstrated that $p s a A$ is upregulated during attachment of pneumococci to nasopharyngeal cells (Marra et al., 2002; Orihuela et al., 2004).

PiaA and PiuA are lipoprotein components of two separate iron-uptake $\mathrm{ABC}$ transporters, and have been shown to be required for full pneumococcal virulence (Brown et al., 2001). Immunization with PiaA and PiuA elicits protective antibodies that recognize these surface-exposed proteins, and recently these antibodies have been demonstrated to promote bacterial opsonophagocytosis rather than inhibit iron transport (Jomaa et al., 2005).

Pneumococci produce two conserved surface-exposed lipoproteins belonging to a family of chaperones, the peptidyl-prolyl isomerases (PPIases), which are thought to be involved in secretion and activation of cell surface molecules. PpmA (putative proteinase maturation protein A) shares homology with members of the parvulin family, 
whereas SlrA shares homology with members of the cyclophilin family. Both lipoproteins have been shown to be immunogenic (Adrian et al., 2004). Involvement in virulence has been suggested for PpmA. Overweg et al. (2000) demonstrated that mutation of ppmA of strain D39 increased the survival rate of mice. SlrA knockout mutants have been shown to be less efficient in the nasopharyngeal colonization of mice, and this has been attributed to the decreased ability of the knockout mutants to adhere to nonprofessional cells (Hermans et al., 2005). Further investigations are required to demonstrate the potential of PpmA and SlrA as vaccine targets.

\section{Moonlighting proteins: keeping the secret}

The surface of S. pneumoniae and other micro-organisms is further decorated by a number of proteins that do not possess classical features of bacterial surface proteins. The PavA protein (pneumococcal adherence and virulence factor A) has been identified as a pneumococcal adhesin for fibronectin and as a crucial virulence determinant in pneumococcal infections. In a systemic and experimental mouse meningitis model of infection, the pneumococcal pavA knockout of strain D39 is substantially attenuated (Holmes et al., 2001; Pracht et al., 2005). The impact of PavA on pathogenesis has been independently indicated by STM experiments using a mouse pneumonia model of infection (Lau et al., 2001). In addition, PavA is most likely involved indirectly and in a fibronectin-independent manner in pneumococcal adherence to host cells. However, the precise function of PavA has yet to be clarified, because deficiency in PavA does not affect the expression and function of known virulence factors (Pracht et al., 2005).

S. pneumoniae acquires host proteolytic activity by binding plasmin(ogen), and interestingly, the glycolytic enzymes enolase and GAPDH have been identified as plasmin(ogen)binding proteins displayed on the cell wall (Bergmann et al., 2004). The moonlighting function of the enolase has been shown to potentiate degradation of the extracellular matrix (ECM), dissolution of fibrin, and pneumococcal transmigration (Bergmann et al., 2005). The key pneumococcal binding site in enolase responsible for plasmin-mediated ECM degradation has been attributed to the nonameric peptide FYDKERKVYD (Bergmann etal., 2003). Crystal structure analysis has indicated an octameric composition of the pneumococcal enolase and has depicted the plasminogenbinding site on the surface of the protein. In contrast, the C-terminal lysine residues, which have previously been suggested to be involved in plasminogen binding, are placed in an interdimer groove and have been predicted to be important for correct folding and for the maintenance of oligomerization (Ehinger et al., 2004). The importance of enolase in virulence has been demonstrated in intranasal infection of mice. Enolase mutants with functionally inactive plasminogen-binding sites are significantly attenuated compared to the isogenic D39 parental strain (Bergmann et al., 2003).

\section{Conclusions and perspectives}

S. pneumoniae produces a broad variety of specialized surface proteins, and it is obvious that at least some of these are adapted to interact with host components during colonization or dissemination in the human host. In contrast to some Gram-negative pathogens, the virulence genes of S. pneumoniae are not generally clustered together. Wholegenome microarray analysis has depicted the differential expression of genes under invasive and non-invasive conditions, and cross-linking of the data with the results of STM and DIF screens provides an extended list of putative virulence factors (Ogunniyi et al., 2002; Orihuela et al., 2004; Marra et al., 2002). These virulence factors can be regulated by one-component regulatory systems, including luxS, rlrA, $r e g M / R$ and $m g r A$, or by one of the 14 TCSs that have been identified in S. pneumoniae (Hoskins et al., 2001; Tettelin et al., 2001). TCSs respond to environmental changes, and therefore mediate the adaptation of pneumococci to their different microenvironments. Throup et al. (2000) and other workers have demonstrated the impact of TCSs on pneumococcal virulence in a mouse infection model. Nevertheless, our current knowledge of the impact of surfaceexposed proteins during specific stages of local and invasive infections caused by pneumococci is fragmentary. In this respect, the individual role of many surface and secreted proteins in pneumococcal virulence remains a mystery. The analysis of crystal structures of surface proteins, alone or in complex with host receptors, can provide detailed molecular insights into the function of these proteins. As virulence is a multifactorial process, a future challenge will be to further elucidate how virulence factors act in concert to determine disease outcome by, for example, sensing the host response. The results of such investigations may clarify the virulence potential of a particular strain and determine which minimal set of factors is required to cause disease.

\section{ACKNOWLEDGEMENTS}

We thank Mark J. Walker (School of Biological Sciences, University of Wollongong, Australia) for critical reading of the manuscript. The work of the group is supported by grants from the German Research Foundation (DFG-SFB 479 to S.H.) and the Federal Ministry of Education and Research (01KI0430 to S. H., Competence Network CAPNETZ). Our apologies in advance to the authors of primary articles whom we have failed to cite owing to space restrictions.

\section{REFERENCES}

Adrian, P. V., Bogaert, D., Oprins, M., Rapola, S., Lahdenkari, M., Kilpi, T., de Groot, R., Kayhty, H. \& Hermans, P. W. (2004). Development of antibodies against pneumococcal proteins alphaenolase, immunoglobulin A1 protease, streptococcal lipoprotein rotamase $\mathrm{A}$, and putative proteinase maturation protein $\mathrm{A}$ in relation to pneumococcal carriage and otitis media. Vaccine 22, 2737-2742.

Bensing, B. A. \& Sullam, P. M. (2002). An accessory sec locus of Streptococcus gordonii is required for export of the surface protein 
GspB and for normal levels of binding to human platelets. Mol Microbiol 44, 1081-1094.

Bergmann, S., Wild, D., Diekmann, O., Frank, R., Bracht, D., Chhatwal, G. S. \& Hammerschmidt, S. (2003). Identification of a novel plasmin(ogen)-binding motif in surface displayed alphaenolase of Streptococcus pneumoniae. Mol Microbiol 49, 411-423.

Bergmann, S., Rohde, M. \& Hammerschmidt, S. (2004). Glyceraldehyde-3-phosphate dehydrogenase of Streptococcus pneumoniae is a surface-displayed plasminogen-binding protein. Infect Immun 72, 2416-2419.

Bergmann, S., Rohde, M., Preissner, K. T. \& Hammerschmidt, S. (2005). The nine residue plasminogen-binding motif of the pneumococcal enolase is the major cofactor of plasmin-mediated degradation of extracellular matrix, dissolution of fibrin and transmigration. Thromb Haemostasis 94, 304-311.

Berry, A. M. \& Paton, J. C. (2000). Additive attenuation of virulence of Streptococcus pneumoniae by mutation of the genes encoding pneumolysin and other putative pneumococcal virulence proteins. Infect Immun 68, 133-140.

Bethe, G., Nau, R., Wellmer, A., Hakenbeck, R., Reinert, R. R., Heinz, H. P. \& Zysk, G. (2001). The cell wall-associated serine protease PrtA: a highly conserved virulence factor of Streptococcus pneumoniae. FEMS Microbiol Lett 205, 99-104.

Blue, C. E., Paterson, G. K., Kerr, A. R., Berge, M., Claverys, J. P. \& Mitchell, T. J. (2003). ZmpB, a novel virulence factor of Streptococcus pneumoniae that induces tumor necrosis factor alpha production in the respiratory tract. Infect Immun 71, 4925-4935.

Brown, J. S., Gilliland, S. M. \& Holden, D. W. (2001). A Streptococcus pneumoniae pathogenicity island encoding an $\mathrm{ABC}$ transporter involved in iron uptake and virulence. Mol Microbiol 40, 572-585.

Cartwright, K. (2002). Pneumococcal disease in western Europe: burden of disease, antibiotic resistance and management. Eur J Pediatr 161, 188-195.

Chapuy-Regaud, S., Ogunniyi, A. D., Diallo, N., Huet, Y., Desnottes, J. F., Paton, J. C., Escaich, S. \& Trombe, M. C. (2003). RegR, a global LacI/GalR family regulator, modulates virulence and competence in Streptococcus pneumoniae. Infect Immun 71, 2615-2625.

Chiavolini, D., Memmi, G., Maggi, T., lannelli, F., Pozzi, G. \& Oggioni, M. R. (2003). The three extra-cellular zinc metalloproteinases of Streptococcus pneumoniae have a different impact on virulence in mice. BMC Microbiol 3, 14.

Cundell, D. R., Gerard, N. P., Gerard, C., Idanpaan-Heikkila, I. \& Tuomanen, E. I. (1995). Streptococcus pneumoniae anchor to activated human cells by the receptor for platelet-activating factor. Nature 377, 435-438.

Dave, S., Carmicle, S., Hammerschmidt, S., Pangburn, M. K. \& McDaniel, L. S. (2004). Dual roles of PspC, a surface protein of Streptococcus pneumoniae, in binding human secretory IgA and factor H. J Immunol 173, 471-477.

De Las Rivas, B., Garcia, J. L., Lopez, R. \& Garcia, P. (2001). Molecular characterization of the pneumococcal teichoic acid phosphorylcholine esterase. Microb Drug Resist 7, 213-222.

Dintilhac, A., Alloing, G., Granadel, C. \& Claverys, J. P. (1997). Competence and virulence of Streptococcus pneumoniae: Adc and PsaA mutants exhibit a requirement for $\mathrm{Zn}$ and $\mathrm{Mn}$ resulting from inactivation of putative ABC metal permeases. Mol Microbiol 25, 727-739.

Duthy, T. G., Ormsby, R. J., Giannakis, E., Ogunniyi, A. D., Stroeher, U. H., Paton, J. C. \& Gordon, D. L. (2002). The human complement regulator factor $\mathrm{H}$ binds pneumococcal surface protein $\mathrm{PspC}$ via short consensus repeats 13 to 15 . Infect Immun 70, 5604-5611.

Ehinger, S., Schubert, W. D., Bergmann, S., Hammerschmidt, S. \& Heinz, D. W. (2004). Plasmin(ogen)-binding alpha-enolase from
Streptococcus pneumoniae: crystal structure and evaluation of plasmin(ogen)-binding sites. J Mol Biol 343, 997-1005.

Elm, C., Braathen, R., Bergmann, S., Frank, R., Vaerman, J. P., Kaetzel, C. S., Chhatwal, G. S., Johansen, F. E. \& Hammerschmidt, S. (2004). Ectodomains 3 and 4 of human polymeric immunoglobulin receptor (hpIgR) mediate invasion of Streptococcus pneumoniae into the epithelium. J Biol Chem 279, 6296-6304.

Garau, G., Lemaire, D., Vernet, T., Dideberg, O. \& Di Guilmi, A. M. (2005). Crystal structure of phosphorylcholine esterase domain of the virulence factor choline binding protein E from Streptococcus pneumoniae: new structural features among the metallo- $\beta$-lactamase superfamily. J Biol Chem 280, 28591-28600.

Gosink, K. K., Mann, E. R., Guglielmo, C., Tuomanen, E. I. \& Masure, H. R. (2000). Role of novel choline binding proteins in virulence of Streptococcus pneumoniae. Infect Immun 68, 5690-5695.

Guiral, S., Mitchell, T. J., Martin, B. \& Claverys, J. P. (2005). Competence-programmed predation of noncompetent cells in the human pathogen Streptococcus pneumoniae: genetic requirements. Proc Natl Acad Sci U S A 102, 8710-8715.

Hammerschmidt, S., Bethe, G., Remane, P. H. \& Chhatwal, G. S. (1999). Identification of pneumococcal surface protein $A$ as a lactoferrin-binding protein of Streptococcus pneumoniae. Infect Immun 67, 1683-1687.

Hammerschmidt, S., Tillig, M. P., Wolff, S., Vaerman, J. P. \& Chhatwal, G. S. (2000). Species-specific binding of human secretory component to SpsA protein of Streptococcus pneumoniae via a hexapeptide motif. Mol Microbiol 36, 726-736.

Hammerschmidt, S., Wolff, S., Hocke, A., Rosseau, S., Muller, E. \& Rohde, M. (2005). Illustration of pneumococcal polysaccharide capsule during adherence and invasion of epithelial cells. Infect Immun 73, 4653-4667.

Hava, D. L. \& Camilli, A. (2002). Large-scale identification of serotype 4 Streptococcus pneumoniae virulence factors. Mol Microbiol 45, 1389-1406.

Hermans, P. W., Adrian, P. V., Albert, C., Estevao, S., Hoogenboezem, T., Luijendijk, I. H., Kamphausen, T. \& Hammerschmidt, S. (2005). The streptococcal lipoprotein rotamase A (SlrA) is a functional peptidyl-prolyl isomerase involved in pneumococcal colonisation. J Biol Chem (in press). http://dx.doi.org/ 10·1074/jbc.M510014200

Hermoso, J. A., Lagartera, L., Gonzalez, A., Stelter, M., Garcia, P., Martinez-Ripoll, M., Garcia, J. L. \& Menendez, M. (2005). Insights into pneumococcal pathogenesis from the crystal structure of the modular teichoic acid phosphorylcholine esterase Pce. Nat Struct Mol Biol 12, 533-538.

Holmes, A. R., McNab, R., Millsap, K. W., Rohde, M., Hammerschmidt, S., Mawdsley, J. L. \& Jenkinson, H. F. (2001). The pavA gene of Streptococcus pneumoniae encodes a fibronectinbinding protein that is essential for virulence. Mol Microbiol 41, 1395-1408.

Hoskins, J., Alborn, W. E., Jr, Arnold, J. \& 39 other authors (2001). Genome of the bacterium Streptococcus pneumoniae strain R6. $J$ Bacteriol 183, 5709-5717.

lannelli, F., Chiavolini, D., Ricci, S., Oggioni, M. R. \& Pozzi, G. (2004). Pneumococcal surface protein C contributes to sepsis caused by Streptococcus pneumoniae in mice. Infect Immun 72, 3077-3080.

Ibrahim, Y. M., Kerr, A. R., McCluskey, J. \& Mitchell, T. J. (2004). Role of HtrA in the virulence and competence of Streptococcus pneumoniae. Infect Immun 72, 3584-3591.

Janulczyk, R., lannelli, F., Sjoholm, A. G., Pozzi, G. \& Bjorck, L. (2000). Hic, a novel surface protein of Streptococcus pneumoniae that interferes with complement function. J Biol Chem 275, 37257-37263. 
Jarva, H., Hellwage, J., Jokiranta, T. S., Lehtinen, M. J., Zipfel, P. F. \& Meri, S. (2004). The group B streptococcal beta and pneumococcal Hic proteins are structurally related immune evasion molecules that bind the complement inhibitor factor $\mathrm{H}$ in an analogous fashion. J Immunol 172, 3111-3118.

Johnson, S. E., Dykes, J. K., Jue, D. L., Sampson, J. S., Carlone, G. M. \& Ades, E. W. (2002). Inhibition of pneumococcal carriage in mice by subcutaneous immunization with peptides from the common surface protein pneumococcal surface adhesin A. J Infect Dis 185, 489-496.

Jomaa, M., Yuste, J., Paton, J. C., Jones, C., Dougan, G. \& Brown, J. S. (2005). Antibodies to the iron uptake $A B C$ transporter lipoproteins PiaA and PiuA promote opsonophagocytosis of Streptococcus pneumoniae. Infect Immun 73, 6852-6859.

Kausmally, L., Johnsborg, O., Lunde, M., Knutsen, E. \& Havarstein, L. S. (2005). Choline-binding protein D (CbpD) in Streptococcus pneumoniae is essential for competence-induced cell lysis. J Bacteriol 187, 4338-4345.

Kharat, A. S. \& Tomasz, A. (2003). Inactivation of the srtA gene affects localization of surface proteins and decreases adhesion of Streptococcus pneumoniae to human pharyngeal cells in vitro. Infect Immun 71, 2758-2765.

King, S. J., Hippe, K. R., Gould, J. M., Bae, D., Peterson, S., Cline, R. T., Fasching, C., Janoff, E. N. \& Weiser, J. N. (2004). Phase variable desialylation of host proteins that bind to Streptococcus pneumoniae in vivo and protect the airway. Mol Microbiol 54, 159-171.

King, S. J., Whatmore, A. M. \& Dowson, C. G. (2005). NanA, a neuraminidase from Streptococcus pneumoniae, shows high levels of sequence diversity, at least in part through recombination with Streptococcus oralis. J Bacteriol 187, 5376-5386.

Lau, G. W., Haataja, S., Lonetto, M., Kensit, S. E., Marra, A., Bryant, A. P., McDevitt, D., Morrison, D. A. \& Holden, D. W. (2001). A functional genomic analysis of type 3 Streptococcus pneumoniae virulence. Mol Microbiol 40, 555-571.

Long, J. P., Tong, H. H. \& DeMaria, T. F. (2004). Immunization with native or recombinant Streptococcus pneumoniae neuraminidase affords protection in the chinchilla otitis media model. Infect Immun 72, 4309-4313.

López, R. \& García, E. (2004). Recent trends on the molecular biology of pneumococcal capsules, lytic enzymes, and bacteriophage. FEMS Microbiol Rev 28, 553-580.

Lu, L., Lamm, M. E., Li, H., Corthesy, B. \& Zhang, J. R. (2003). The human polymeric immunoglobulin receptor binds to Streptococcus pneumoniae via domains 3 and 4. J Biol Chem 278, 48178-48187.

Luo, R., Mann, B., Lewis, W. S. \& 9 other authors (2005). Solution structure of choline binding protein $\mathrm{A}$, the major adhesin of Streptococcus pneumoniae. EMBO J 24, 34-43.

Marra, A., Lawson, S., Asundi, J. S., Brigham, D. \& Hromockyj, A. E. (2002). In vivo characterization of the psa genes from Streptococcus pneumoniae in multiple models of infection. Microbiology 148, 1483-1491.

Mascher, T., Zahner, D., Merai, M., Balmelle, N., de Saizieu, A. B. \& Hakenbeck, R. (2003). The Streptococcus pneumoniae cia regulon: CiaR target sites and transcription profile analysis. J Bacteriol 185, 60-70.

Ng, W. L., Tsui, H. C. \& Winkler, M. E. (2005). Regulation of the $p s p A$ virulence factor and essential $p c s B$ murein biosynthetic genes by the phosphorylated VicR (YycF) response regulator in Streptococcus pneumoniae. J Bacteriol 187, 7444-7459.

Oggioni, M. R., Memmi, G., Maggi, T., Chiavolini, D., lannelli, F. \& Pozzi, G. (2003). Pneumococcal zinc metalloproteinase ZmpC cleaves human matrix metalloproteinase 9 and is a virulence factor in experimental pneumonia. Mol Microbiol 49, 795-805.

Ogunniyi, A. D., Giammarinaro, P. \& Paton, J. C. (2002). The genes encoding virulence-associated proteins and the capsule of Streptococcus pneumoniae are upregulated and differentially expressed in vivo. Microbiology 148, 2045-2053.

Orihuela, C. J., Radin, J. N., Sublett, J. E., Gao, G., Kaushal, D. \& Tuomanen, E. I. (2004). Microarray analysis of pneumococcal gene expression during invasive disease. Infect Immun 72, 5582-5596.

Overweg, K., Kerr, A., Sluijter, M., Jackson, M. H., Mitchell, T. J., de Jong, A. P., de Groot, R. \& Hermans, P. W. (2000). The putative proteinase maturation protein A of Streptococcus pneumoniae is a conserved surface protein with potential to elicit protective immune responses. Infect Immun 68, 4180-4188.

Paterson, G. K. \& Mitchell, T. J. (2005). The role of Streptococcus pneumoniae sortase A in colonisation and pathogenesis. Microbes Infect (in press).

Paterson, G. K. \& Mitchell, T. J. (2006). Innate immunity and the pneumococcus. Microbiology 152, 285-293.

Polissi, A., Pontiggia, A., Feger, G., Altieri, M., Mottl, H., Ferrari, L. \& Simon, D. (1998). Large-scale identification of virulence genes from Streptococcus pneumoniae. Infect Immun 66, 5620-5629.

Pracht, D., Elm, C., Gerber, J. \& 7 other authors (2005). PavA of Streptococcus pneumoniae modulates adherence, invasion, and meningeal inflammation. Infect Immun 73, 2680-2689.

Quin, L. R., Carmicle, S., Dave, S., Pangburn, M. K., Evenhuis, J. P. \& McDaniel, L. S. (2005). In vivo binding of complement regulator factor $\mathrm{H}$ by Streptococcus pneumoniae. J Infect Dis 192, 1996-2003.

Ren, B., McCrory, M. A., Pass, C., Bullard, D. C., Ballantyne, C. M., Xu, Y., Briles, D. E. \& Szalai, A. J. (2004). The virulence function of Streptococcus pneumoniae surface protein A involves inhibition of complement activation and impairment of complement receptormediated protection. J Immunol 173, 7506-7512.

Rigden, D. J. \& Jedrzejas, M. J. (2003). Structures of Streptococcus pneumoniae hyaluronate lyase in complex with chondroitin and chondroitin sulfate disaccharides. Insights into specificity and mechanism of action. J Biol Chem 278, 50596-50606.

Romero-Steiner, S., Pilishvili, T., Sampson, J. S., Johnson, S. E., Stinson, A., Carlone, G. M. \& Ades, E. W. (2003). Inhibition of pneumococcal adherence to human nasopharyngeal epithelial cells by anti-PsaA antibodies. Clin Diagn Lab Immunol 10, 246-251.

Rosenow, C., Ryan, P., Weiser, J. N., Johnson, S., Fontan, P., Ortqvist, A. \& Masure, H. R. (1997). Contribution of novel cholinebinding proteins to adherence, colonization and immunogenicity of Streptococcus pneumoniae. Mol Microbiol 25, 819-829.

Sebert, M. E., Palmer, L. M., Rosenberg, M. \& Weiser, J. N. (2002). Microarray-based identification of htrA, a Streptococcus pneumoniae gene that is regulated by the CiaRH two-component system and contributes to nasopharyngeal colonization. Infect Immun 70, 4059-4067.

Shakhnovich, E. A., King, S. J. \& Weiser, J. N. (2002). Neuraminidase expressed by Streptococcus pneumoniae desialylates the lipopolysaccharide of Neisseria meningitidis and Haemophilus influenzae: a paradigm for interbacterial competition among pathogens of the human respiratory tract. Infect Immun 70, 7161-7164.

Shaper, M., Hollingshead, S. K., Benjamin, W. H., Jr \& Briles, D. E. (2004). PspA protects Streptococcus pneumoniae from killing by apolactoferrin, and antibody to PspA enhances killing of pneumococci by apolactoferrin. Infect Immun 72, 5031-5040.

Takamatsu, D., Bensing, B. A. \& Sullam, P. M. (2004). Genes in the accessory sec locus of Streptococcus gordonii have three functionally 
distinct effects on the expression of the platelet-binding protein GspB. Mol Microbiol 52, 189-203.

Tettelin, H. \& Hollingshead, S. K. (2004). Comparative genomics of Streptococcus pneumoniae: intrastrain diversity and genome plasticity. In The Pneumococcus, pp. 15-29. Edited by E. I. Tuomanen, T. J. Mitchell, D. A. Morrison \& B. G. Spratt. Washington, DC: American Society for Microbiology.

Tettelin, H., Nelson, K. E., Paulsen, I. T. \& 36 other authors (2001). Complete genome sequence of a virulent isolate of Streptococcus pneumoniae. Science 293, 498-506.

Throup, J. P., Koretke, K. K., Bryant, A. P. \& 9 other authors (2000). A genomic analysis of two-component signal transduction in Streptococcus pneumoniae. Mol Microbiol 35, 566-576.

Tong, H. H., Liu, X., Chen, Y., James, M. \& Demaria, T. (2002). Effect of neuraminidase on receptor-mediated adherence of Streptococcus pneumoniae to chinchilla tracheal epithelium. Acta Otolaryngol 122, 413-419.

Tseng, H. J., McEwan, A. G., Paton, J. C. \& Jennings, M. P. (2002). Virulence of Streptococcus pneumoniae: PsaA mutants are hypersensitive to oxidative stress. Infect Immun 70, 1635-1639.

Vollmer, W. \& Tomasz, A. (2001). Identification of the teichoic acid phosphorylcholine esterase in Streptococcus pneumoniae. Mol Microbiol 39, 1610-1622.

Weiser, J. N., Bae, D., Fasching, C., Scamurra, R. W., Ratner, A. J. \& Janoff, E. N. (2003). Antibody-enhanced pneumococcal adherence requires IgA1 protease. Proc Natl Acad Sci U S A 100, 4215-4220.

Zhang, J. R., Mostov, K. E., Lamm, M. E., Nanno, M., Shimida, S., Ohwaki, M. \& Tuomanen, E. (2000). The polymeric immunoglobulin receptor translocates pneumococci across human nasopharyngeal epithelial cells. Cell 102, 827-837. 